\title{
Improvement of Chronic Rhinitis Under Aspirin
}

\author{
Christos Merkonidis MD, Volker Gudziol MD, Mariapaola Paola Cecchini MD, \\ Johannes Gerber MD, Han-Seok Seo MD, Claus Bachert MD PhD, \\ and Thomas Hummel MD
}

\begin{abstract}
In a subset of aspirin-tolerant asthmatics, administration of aspirin improves respiratory symptoms. We present a patient with chronic rhinosinusitis with nasal polyps who exhibited relief of nasal obstruction and nasal discharge and improvement in the sense of smell following oral administration of $150 \mathrm{mg}$ of aspirin daily. Improvement in the patency of the nasal passages was documented by nasal endoscopy and magnetic resonance imaging. Improvement of olfactory function was documented by validated psychophysical olfactory testing and by means of olfactory event-related potentials. Key words: aspirin; salicylic acid; olfaction disorders; sinusitis; nasal polyps. [Respir Care 2012;57(3):460-463. (C) 2012 Daedalus Enterprises]
\end{abstract}

\section{Introduction}

In contrast to aspirin-exacerbated respiratory disease, there are some reports about patients in whom asthma improves with aspirin, although those reports provide few data on aspirin's impact on chronic rhinosinusitis or olfaction. ${ }^{1-3}$

\section{Case Report}

We saw a 54-year-old non-smoking male, with a 3-year history of fluctuating sense of smell, profuse rhinorrhea,

\footnotetext{
Dr Merkonidis is affiliated with the Department of Otorhinolaryngology, Ipswich Hospital, Ipswich, United Kingdom. Drs Gudziol, Cecchini, Seo, and Hummel are affiliated with the Smell and Taste Clinic, Department of Otorhinolaryngology, University of Dresden Medical School, Dresden, Germany. Dr Gerber is affiliated with the Department of Neuroradiology, University of Dresden Medical School, Dresden, Germany. Dr Bachert is affiliated with the Upper Airways Research Laboratory, Neus, Keel, Oren (Ear, Nose, and Throat) Department, University Hospital Ghent, Ghent, Belgium. Dr Cecchini is also affiliated with the Section of Anatomy and Histology, Department of Morphological and Biomedical Sciences, University of Verona Medical School, Verona, Italy.
}

The authors have disclosed no conflicts of interest.

Correspondence: Volker Gudziol MD, Smell and Taste Clinic, Department of Otorhinolaryngology, University of Dresden Medical School, Fetscherstrasse 74, 01307 Dresden, Germany. E-mail: volker.gudziol@ uniklinikum.dresden.de.

DOI: $10.4187 /$ respcare. 01190 and nasal obstruction. Since he had started taking $150 \mathrm{mg}$ of aspirin per day to prevent atherosclerosis, he observed an improvement of his sense of smell and a decrease of rhinorrhea and nasal obstruction. After taking aspirin in the morning his sense of smell would improve and be normal around lunchtime and would then gradually deteriorate in the afternoon. He had a history of essential hypertension, for which he was on felodipine $5 \mathrm{mg}$ and ramipril $5 \mathrm{mg}$ daily. He reported bronchial hyper-responsiveness to volatile chemicals, but he did not have asthma. Allergy skin prick testing to common aeroallergens was negative.

We tested for chronic rhinosinusitis and olfactory function on 3 different days. On day 1 he took aspirin in the morning, and the olfaction tests were performed at least 4 hours after aspirin ingestion. The next day (day 2) he did not take aspirin until all the tests were completed. On day 3 and day 4 he took aspirin in the morning. He had been on aspirin for 44 hours when the final test was performed, but at least 7 hours after his last aspirin dose.

Figure 1 shows images from nasal endoscopy on aspirin and off aspirin. Figure 2 shows magnetic resonance images on aspirin, at 29 hours off aspirin, and 44 hours after resuming aspirin.

We assessed olfaction with the validated Sniffin' Sticks test kit (Table 1). ${ }^{4}$ We measured olfactory event-related potentials with an air-dilution olfactometer (OM2S, Burghart, Wedel, Germany) with cinnamon aldehyde $(20 \% \mathrm{v} / \mathrm{v})$ stimuli, with a duration $200 \mathrm{~ms}$ and an inter- 


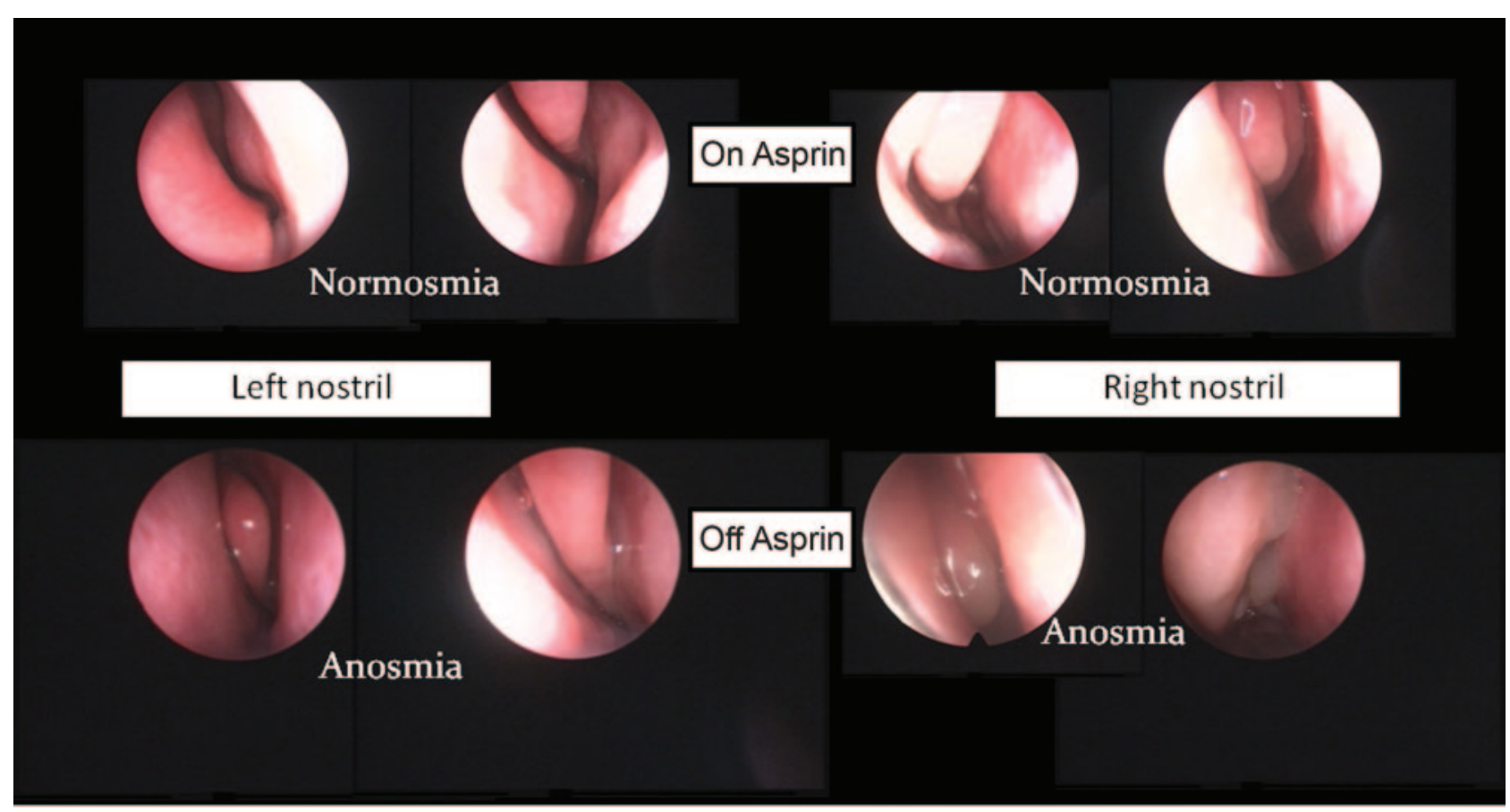

Fig. 1. Nasal cavities on aspirin (upper row) and off aspirin (lower row). In this patient, on aspirin the left side is decongested and dry and the right side, which has a polyp in the olfactory cleft, is fairly decongested and wet. Off aspirin the mucosa showed marked edema and clear nasal discharge on the right, and the left side is congested and wet.
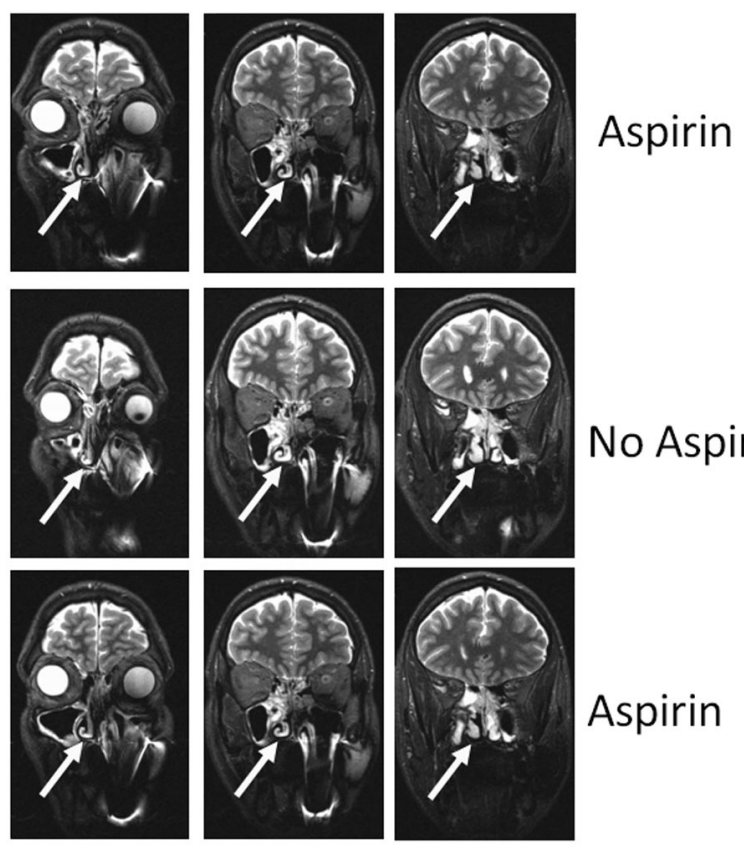

No Aspirin

Fig. 2. Magnetic resonance images (T2-weighted, coronal slices) on aspirin (upper row) after 29 hours off aspirin (middle row), and 44 hours after resuming aspirin (bottom row). The arrow indicates the right inferior turbinate. On aspirin the turbinate's mucosa is less swollen than it is off aspirin. There are no major morphological changes on either side of the olfactory cleft.

stimulus interval $30 \mathrm{~s}$, in a constant stream of humidified air (Fig. 3). ${ }^{5}$
Table 1. The TDI Scores While on Aspirin, When 29 Hours Off Aspirin, and 44 Hours After Resumption of Aspirin, and the Corresponding Scores

\begin{tabular}{lccc}
\hline \hline On Aspirin & $\begin{array}{c}\text { 29 Hours } \\
\text { Off Aspirin }\end{array}$ & $\begin{array}{c}\text { 44 Hours } \\
\text { After Resumption } \\
\text { of Aspirin }\end{array}$ \\
\hline Threshold & 5 & 1 & 3 \\
Discrimination & 13 & 5 & 14 \\
Identification & 14 & 9 & 15 \\
Total TDI Score & 32 & 15 & 32 \\
& & & \\
TDI = threshold, discrimination, identification & & \\
\hline
\end{tabular}

\section{Discussion}

There have been few reports, mainly from the late 1970s and 1980s, of aspirin-tolerant asthmatics who experienced bronchodilatation from aspirin or other cyclooxygenase inhibitors. ${ }^{1-3,6,7}$ The benefit was reported to occur within $30-60$ min after of aspirin ingestion, and to last several hours, and in one case 3 days. ${ }^{3}$ Symptom improvement was observed only with cyclooxygenase inhibitors, and it was hypothesized that the effect was caused by a diversion of the arachidonic acid metabolism toward cyclooxygenase products. ${ }^{2}$

Our patient did not have asthma but did report bronchial hyper-responsiveness to volatile chemicals. Lung-function tests on and off aspirin would be desirable, but our patient 

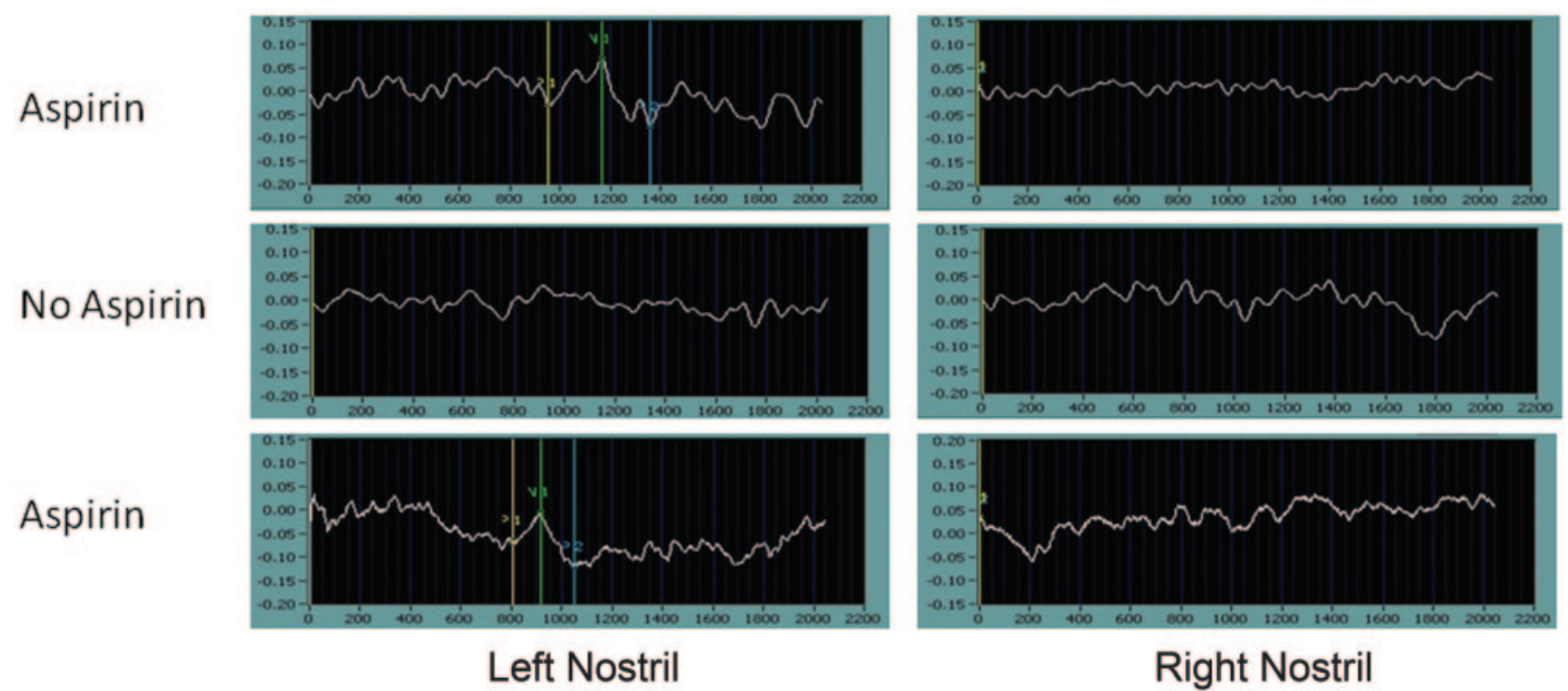

Right Nostril

Fig. 3. Olfactory event-related potentials while on aspirin, off aspirin, and 44 hours after resuming aspirin (top). Olfactory event-related potentials could only be obtained from the left nostril while on aspirin. Off aspirin no responses could be obtained from either side. Response peaks are indicated by markers. Stimulus onset starts at $530 \mathrm{~ms}$ after onset of recordings.

declined lung function testing, because he did not feel any respiratory impairment or lung-function difference on versus off aspirin. He did accede to nasal endoscopy, magnetic resonance imaging, and olfaction testing. While he was on aspirin he was normosmic, 29 hours off aspirin he was anosmic, and olfactory function had returned to normal 44 hours after restarting aspirin (see Table 1). Olfactory event-related potentials could be obtained while the patient was on aspirin, but could not be obtained when the patient was off aspirin for 29 hours. Polyps in the right olfactory cleft that prevented air from reaching the olfactory receptors might explain why olfactory event-related potentials could be obtained only from the left nostril. Birhinally-obtained olfactory test results by means of the Sniffin' Sticks test represent olfactory function of the better nostril. That is why it is possible that he had irrecoverable loss of olfaction on the right side, ${ }^{8}$ but the absence of rightside olfaction might have been from nasal congestion that might have been reversed by a higher dose of aspirin. ${ }^{9}$

There have been no broad-based epidemiological studies on the prevalence of aspirin-relieved respiratory disease. The available data suggest a prevalence of $0.5-2 \%$ among asthmatic patients. ${ }^{3,7,10}$ Upper-respiratory-tract inflammation and chronic rhinosinusitis frequently reduce olfaction and decrease quality of life,,$^{11-13}$ and the available therapies are limited and their effects transitory. ${ }^{14,15} \mathrm{~A}$ study is needed on whether aspirin-tolerant patients with chronic rhinosinusitis and/or asthma benefit from ingested or topical aspirin. ${ }^{16}$ Topical lysine aspirin desensitizes aspirin-exacerbated respiratory disease, and has been reported to be safe and suitable for long-term use. ${ }^{17}$ Some patients with olfactory loss due to chronic rhinosinusitis, with or without nasal polyps, might benefit from aspirin.

\section{REFERENCES}

1. Imokawa S, Sato A, Taniguchi M, Toyoshima M, Hayakawa H, Chida K. Lipoxygenase inhibitor-provoked acute asthma in a patient with asthma relieved by aspirin. Ann Allergy Asthma Immunol 1995; 75(2):112-114.

2. Garin PR, Frans A. Aspirin-relieved asthma. Med Hypotheses 1990; 32(2):125-128.

3. Resta O, Foschino-Barbaro MP, Carnimeo N, Bavoso P, Picca V. Asthma relieved by acetylsalicylic acid and nonsteroid anti-inflammatory drugs. Respiration 1984;46(1):121-127.

4. Hummel T, Kobal G, Gudziol H, Mackay-Sim A. Normative data for the "Sniffin' Sticks" including tests of odor identification, odor discrimination, and olfactory thresholds: an upgrade based on a group of more than 3,000 subjects. Eur Arch Otorhinolaryngol 2007;264(3): 237-243.

5. Hummel T, Welge-Luessen A. Assessment of olfactory function. Adv Otorhinolaryngol 2006;63:84-98.

6. Szczeklik A, Gryglewski RJ, Nizankowska E. Asthma relieved by aspirin and by other cyclo-oxygenase inhibitors. Thorax 1978;33(5): 664-665.

7. Kordansky D, Adkinson NF, Jr., Norman PS, Rosenthal RR. Asthma improved by nonsteroidal anti-inflammatory drugs. Ann Intern Med 1978;88(4):508-511.

8. Klimek L, Hummel T, Moll B, Kobal G, Mann WJ. Lateralized and bilateral olfactory function in patients with chronic sinusitis compared with healthy control subjects. Laryngoscope 1998;108(1 Pt 1):111-114

9. Rozsasi A, Polzehl D, Deutschle T, Smith E, Wiesmiller K, Riechelmann $\mathrm{H}$, et al. Long-term treatment with aspirin desensitization: a 


\section{IMPROVEMENT OF CHRONIC RHINITIS UNDER ASPIRIN}

prospective clinical trial comparing 100 and $300 \mathrm{mg}$ aspirin daily. Allergy 2008;63(9):1228-1234.

10. Pearson R. Hypersensitivity to aspirin. In: Dixon A, Martin B, Smith M, Wood P, editors. Salicylates. Boston: Brown and Company; 1963: 170-173.

11. Miwa T, Furukawa M, Tsukatani T, Costanzo RM, DiNardo LJ, Reiter ER. Impact of olfactory impairment on quality of life and disability. Arch Otolaryngol Head Neck Surg 2001;127(5):497-503.

12. Gudziol V, Wolff-Stephan S, Aschenbrenner K, Joraschky P, Hummel T. Depression resulting from olfactory dysfunction is associated with reduced sexual appetite: a cross-sectional cohort study. J Sex Med 2009;6(7):1924-1929.

13. Konstantinidis I, Witt M, Kaidoglou K, Constantinidis J, Gudziol V. Olfactory mucosa in nasal polyposis: implications for FESS outcome. Rhinology;48(1):47-53.
14. Blomqvist EH, Lundblad L, Bergstedt H, Stjarne P. Placebo-controlled, randomized, double-blind study evaluating the efficacy of fluticasone propionate nasal spray for the treatment of patients with hyposmia/anosmia. Acta Otolaryngol 2003;123(7):862-868.

15. Gudziol V, Buschhuter D, Abolmaali N, Gerber J, Rombaux P, Hummel T. Increasing olfactory bulb volume due to treatment of chronic rhinosinusitis: a longitudinal study. Brain 2009;132(Pt 11): 3096-3101.

16. Nucera E, Schiavino D, Milani A, Del Ninno M, Misuraca C, Buonomo A, et al. Effects of lysine-acetylsalicylate (LAS) treatment in nasal polyposis: two controlled long term prospective follow up studies. Thorax 2000;55(Suppl 2):S75-S78.

17. Parikh AA, Scadding GK. Intranasal lysine-aspirin in aspirin-sensitive nasal polyposis: a controlled trial. Laryngoscope $2005 ; 115(8)$ : 1385-1390. 